\title{
Uniqueness, Self belonging and Intercourse in Nature
}

Marvin E. Kirsh

ISBN: 3-8383-6737-8

Lambert Academic Publishing

Saarbrücken, Germany

Prologue and Preface

Prologue

\section{Trepidations of a Dancer}

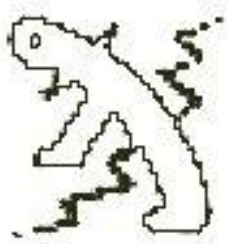


Is it wisdom

or isnt it fact

to say for fact

that a lighted path

takes its meaning of the dark

dark takes its meaning of the light?

suffer one a cold dark matter

a warm shade of light

if one there is the other

warm fuel cold fuel

cold fuel warm fuel

A full fueled fool

fulfills the whole infernal eternio

stayed in the dark

a fools measured feet

full measured foot

stays the whole path

cold foot

warm foot

5280 feet 
186,000 miles per second

Exon, Amoco,

Van Goughs Ceiling Senerio

The telescope on Mount Palamar

Fifty Scholastic Seminars

A bank of neuroeconomic bills

light cold warm dark

amount of physical matter

a mount of energy

A slow or fast courtship

a'mate different shadows

in the dance

what is

what lasts

Marvin E. Kirsh 



\section{Preface}

Conceptual understanding of the environment, in first reflexive moments, entails strings of cause and effect, both in ordinary life and in extended scientific analysis. In this sense, the layman and the scientist really harbor no difference in their professional qualifications with which to obtain license to profess about the realities of life, though the scientist not only forms abstractions and conjectures concerning parts of the world that are entailed, from his definitions to be strictly unwitnessible, but constructs tools to extend the power of his senses. It is not even unfeasible that the ordinary person is able to build similar spying devices that can have applications extending from the ordinary social aspects of life to the grand notions involving politics and military in order to obtain knowledge of events that are beyond his living sphere; it would, however be very unlikely for a reflecting human social being to abstract into existence unwitnessible entities for explanation and application to understanding of problems and events of personal, social or political existence, especially for everyday use.

The scientist, in order to construct notions, has entered into the occupation of building rationalized symbolisms made of the most fundamental of mathematical operations, additions, that are founded from within the same first universal and historically propagated reflexive language involving the never ending open of the world in the form of the expression 'infinity' and a corresponding mathematical symbol that is given a rationally chosen set of appropriate operations to be used in corresponding relations. Mathematical languages, though, are not final no matter how tight their logic, but are only expressions, descriptions created by a subset of the total of life and survival practitioners-i.e. all men and women are practitioners of the same science of life and survival. 
Peculiar to the ubiquitous practice of life, even its' empirical investigation by experts in the life sciences professions, biologists, is a historically propagated concept, especially prominent in the physical scientific sector, of a universe that originates with inert materials from which life is born-i.e. birth in a step wise fashion of cause and effect, pursues death through the perceived vast unending open of the spaces of the universe in which energy is utilized to exhaustion towards the accomplishment of an unwitnessible end: birth and death, both unwitnessible, are the central topics of the speculation that common sense laws of energy and matter can be applied to the construction of a universal mathematical theory to explain the processes of the universe in an ordered and comprehendible fashion. Scientific views, at the roots of their founding logic and paradigms, though argued over, have an innate affinity to all practitioners as they are based equally on the same logic employed by all to navigate the world; general acceptance of abstractions employed by the scientist entail also the acceptance of the scientists philosophy and method that is not generally expressed or put to the same scrutiny and method of comparison as theory contents ; it is eccentrically goal oriented in a direction that is detoured infinitely from potential questions that may arise about its' nature from self examination and, on a self created, finite for comprehension, path in which scrutiny is not upon itself and its' own symbolism but upon the content of theory. External nature (re)defined this way may unconsciously entail, rather than man as finite and nature as infinite in a world that can continue without him, an opposite view of man himself, his ideas as infinite and nature finite. If one takes a few strides along this path he might perceive that scientific progress entails the simultaneous reduction of both man and nature, as men are constructed of the elements of nature. In further reflection, if nature is defined to be space and volume, whatever its' contents, they are also spaces and volumes, a parallel devised with respect to criteria of finite or infinite, mortal or 
immortal, between 'the concept' as it is construed as a tool in science whose character is beyond its' own scrutiny and a possible tangible knowing able to gives it a finite character, potentially emerged from it and a similar parallel evolved between the scientific knowing of volumes of space and a conceptual character to the physical volume is a physical nature with respect to a concept that can relate the concept of volume to 'the concept' itself, -i.e. a concept of 'the concept' that physical/volumetric in nature. Though this may sound to be a far fetched abstraction arising at the extreme end of the scale of what might be considered feasible for abstractions. If it is only the physical volume of nature from which all life experience, all thinking and concepts originate, is the physical means of functioning of the biological material, the mind and body that create concepts, and the activities of cognition and conceptualization are a survival priority endowment, i.e.-the world as an outfit of ordered and ordering associations arising from and molded to the nature of the physical environment, then implied is a notion of the identity possessing 'concept' as a particular that is specifically entangled with the physical path of the entity that is itself a manifestation of path and potentially characterized in a corresponding physical manner of some sort as are other manifestations of path in the sciences that are constructed in terms that are familiar to perception.

In order to assemble applications to the sciences of living, the space and room sciences of the world, in attempts to conserve enormous life time and effort to focus the intellect on what is directly within physical grasp, abstracted attempts to capture the intangible concepts of time, infinity, etc. should necessarily be detoured from the first bird in hand, the laws of energy and motion, to assume that all, either within direct reach of sensory experience or beyond, is of the same physical nature as the coordinated senses indicate of the immediate environment. If all that is conceivable is a matter of mind, as it is the mind that conceives yet escapes a tangible 
comprehension of its' own existence, in a world of accelerating high tech crime, an unexpected and scrupulously honest and extensively brief and vast labor shortcut may exist with the premise that all is a matter of material matter whose birth is not the result of a construction effort but of a failed destruction, that it is death that precedes and pursues life.

The subject of this manuscript is modern scientific effort. If it focused upon the line of cause and effect, if mankind seeks to know his own line from an elaborated chain of cause and effect, perhaps he does not, for survival, need to elaborate the last details of observation to extend himself looking for evidence in a scheme of his own similar making, to beyond what is logically witnessible for facts to satisfy the entire set of plausible questions, but to ask more pertinently about the physical line, volume, and hence life, life and survival might be accounted in physical terms, in familiar physical units.

Uniqueness is common to all of the worlds descriptions, no two are exactly identical. Description, belongs to the set of descriptions, is self belonging as a (descriptive) set of descriptions; the content of the set of descriptions can emerge but it is not logical to assume that the descriptor, 'description' itself can emerge, it serves as door stop to a man-contrived passage between the physical particulars of nature and their descriptions. A unique set of descriptions of necessarily unique items from nature, necessarily unique, is self belonging, where as a description of the physical entity is not a physical entity, is not suitable for numerical parametric description; content leading beyond the apparent physically existing should be limited to the set of unique descriptions-i.e. an electron should not be given a mass value if it results, e.g. in a gravitational field, to have physically testified qualities; one cannot deduce from the precipice of induction without relocating the frame of experiment exclusively away from potential witness and thus from potential appropriateness to all witness. It is this problem that leads to the 
frustration to define inertial frames, especially when constants are involved as they consistently refer to an 'everywhere' that is necessarily elsewhere, and away from actual priorities. If the world can be viewed as self belonging conceptually, and the same in parallel as a physical volume, composed of physical volumes, it might be reduced, holistically, though admittedly with imagination, to a single self belonging set. It should not, in first reflex, be named as physical and conceptual/descriptive, physical and/or conceptual, but technically, logically, exclusively, as physical; though all knowledge involves the mind, it originates exclusively with the physical.

It is from this premise that a tangible connection is meant to be established between what is physical/ corporeal, what is conceptual/incorporeal, and the property of uniqueness and self belonging. In the path of a reduction of nature as an entity of mind and matter to an exclusive entity of physical containers and contents, it is proposed, in a scientifically unholy manner, though not without physical evidence, that nature, relative nature, absent of standards and constants, keeps time in every corner without exception, as a clock whose hands are never at the same position. The unwitnessible products of occurring processes in necessarily unwitnessible containers birth all the things that are basic, evident and prominent. One cannot witness himself, his own culture, but to describe some facets of himself/his culture.

'The concept' is discussed as an entity of the activity occurring in the volumes of space that contain the environment, itself the contents of a volume that is beyond witness and is responsible for both the structure of the physical environment and hence the structure of concepts assembled at the position of witness. The physical environment and the conceptual outfit are mutually dependent properties of both their own structure and function and the properties of unwitnessible volumes that physically contain them. Thus the properties of the earth and its' associated environment, as heterogeneous entities composed of heterogeneous entities, are 
dependent on their interactions over time, beginning from the forces responsible for their births, with the volumes of space they are associated with, and so on, whatever the associations of those volumes. The concept is given a simple description as an unwitnessible product of the interaction of volumes, of volumes containing volumes. A box of red light and a box of blue light in proximity are suggested to birth a proximity dependent concept of color that is a product of nature and the consequence of the propagation of inherited proximities, etc. Concepts are postulated likewise to similarly give birth to cognitive ability as the interaction of proximally inherited conceptual states that arise from the perceptual sensing of physical interactions over time, with generations. Both the world and the modern scientifically essential interval of time in the chain of cause and effect, are better understood in a scheme of mirrors, symbolism and need, though filled with time, time is not considered empirically but as a concept that is a function of many variables. A world construct is proposed that is purged of both quantitative ideations of time, and an absolute necessity for an order of events.

As an example of the possible equivalence of the path dependent physical and conceptual, a conceptual model for the elaboration of a shape for space is given that is derived from the projection of light emitted from a linear motion with an expenditure of energy and the accumulation of mass that is accounted for by a loss of velocity. Magnitudes of ordinary motion and lengths, predicted from the special theory of relativity and a decrease in velocity of light with the expenditure of energy combined with Newtons laws of motion, generated from independent angular parameters, produce an exact replica of the ordinary egg found in nature. It is proposed that the nature of the universe is such that change to the energies of fast electromagnetic radiation, stripped of temporal components and translated into lengths, coincide with the ranges of ordinary perceptions that are likewise striped of temporal components: 
considered this way, slow and fast motion in a synergy, define the realm of all observation as a mirrored relation of a relation of mirrors, and form the physical world.

Though it is evident that the world is composed strictly of energy, it is a matter of preference that it is referred to as matter; regardless of which preceded either, the popularly pursued topic of a structure of matter is more effortlessly ordered both conceptually and physically than a relativity of energy relations is ordered conceptually. Suggested to emerge from the conceptual divide of (conceptually and physically ordered) matter and (conceptually) ordered energy is 'the concept', a concept of the physical as a breeching, proposed to be, in the extended temporal frame of civilization, from its' start to present, the actual and single topic in the history of both mankinds' intellectual-conceptual and physical pursuits. It is the divide (of energy and matter) that is always pursued in a stationery focus. In analogy, a study of the parameters of ice melting to water in a container is attached universal significance in the form of constants that in reality bear no universal significance in a conceptual frame defined by divides that are born only from an existing vast diversity of parametric incongruities of tempos and rhythms, and lead to witness encounters as a result of a past dictated happenstance in the dynamically evolving proximities of agents. As singular and appropriate definition, the world is defined to be ordered from its' root as a physical breaching of the physical from which both mind and matter are derived and are accounted comprehensively to 'the concept'; 'the physical concept' gives explanation for that which fills the divide, as the ether, as a medium that is occupied by a geometry of mirrors, mirrored proximities of mirrors, of mirrors of mirrors of physical volumes that is unable to witness or know simultaneously both it's own reflection and a reflection of its' own reflection, is occupied living out the only logically possible birth in the direction of death. 
I hope to stress that man as a part of nature is an entity of its' processes, processes that in the acts of creativity are unique, represent or not uniqueness within the creative interpretation of men seeking explanation. Man has more and more come to admire in his works the feature of self definition as it resembles nature, employing an appearance of it as judgment criteria in tests of validity. The creative creations of men need not necessarily fall into a set that accommodates the actual path of nature, but perhaps only a branch of a whole course that modulates all events; interpretation from a first perspective of men on the earth maybe time-ofobservation related and inapplicable for the creation of theories with an ultimate goal related to the satisfaction of human needs over an extended range of time. As the productions of mankind come to bear life, the flows and processes, if not life and its' components tapped to reform the environment, relying on distant abstracted perspectives that are removed from immediate experience, embedded with principle notions involving a conceptual stationery aspect, voluntarily remove the first person from the actual perspective, and are not, though self created (excuse the pun as they neither contain themselves or refer to a locus that can be defined as the perspective of mankind), self belonging-i.e. belong to the same set that contains themselves; the set of the works of Karl Marx is not a member of the set of the works of Karl Marx but of the set of human creations that in turn can be said to be a finite and particular fact of history that is itself, whether totally comprised of the activities of men, not a human creation but an occurrence. In contrast, physical and conceptual spaces, proposed to both be ultimately of a physical nature related to physical spaces, apriorily belong to both themselves and the self as they define both the self and themselves, are self belonging. The worlds energy is embodied to the existence and complexity of 3-D form rather than to lines of cause and effect: a correct ordering of nature that leans away from endeavors strictly influenced from ideations of birth and 
death, time, to a simple physical ordering based on physical shape and geometry, as the world is actually perceived, is presented and considered to be of paramount priority. Two conditions are present to the experience of nature, energy bound to form as well as form bound to energy. 